\title{
Interactive effects of the probability of the cue and the probability of the outcome on the overestimation of null contingency
}

\author{
Fernando Blanco • Helena Matute - Miguel A. Vadillo
}

Published online: 26 March 2013

(C) Psychonomic Society, Inc. 2013

\begin{abstract}
Overestimations of null contingencies between a cue, $\mathrm{C}$, and an outcome, $\mathrm{O}$, are widely reported effects that can arise for multiple reasons. For instance, a high probability of the cue, $P(\mathrm{C})$, and a high probability of the outcome, $P(\mathrm{O})$, are conditions that promote such overestimations. In two experiments, participants were asked to judge the contingency between a cue and an outcome. Both $P(\mathrm{C})$ and $P(\mathrm{O})$ were given extreme values (high and low) in a factorial design, while maintaining the contingency between the two events at zero. While we were able to observe main effects of the probability of each event, our experiments showed that the cue- and outcome-density biases interacted such that a high probability of the two stimuli enhanced the overestimation beyond the effects observed when only one of the two events was frequent. This evidence can be used to better understand certain societal issues, such as belief in pseudoscience, that can be the result of overestimations of null contingencies in high$P(\mathrm{C})$ or high- $P(\mathrm{O})$ situations.
\end{abstract}

Keywords Causal learning · Contingency judgment

It is a common assumption that certain human cognitive abilities are grounded in the accurate perception of statistical contingency between variables. A celebrated example is causal learning and inference, which constitutes the major topic of this report. The philosopher David Hume (1739/1987) stressed the relevance of contingency in causal inference, as

F. Blanco $(\bowtie) \cdot$ H. Matute

Departamento de Fundamentos y Métodos de la Psicología,

University of Deusto, Apdo. 1,

48080 Bilbao, Spain

e-mail: fblanco81@gmail.com

M. A. Vadillo

Division of Psychology and Language Sciences, University

College London, London, UK follows: Other factors held constant, effects must be contingent on their causes. That is, the occurrence of the cause should alter the probability of the effect occurrence. Hence, this invariance can be exploited by human and nonhuman animals to infer causality from their exposure to contingency patterns. In line with this view, prominent theories and empirical reports (Rescorla, 1968; Wasserman, 1990) have supported the hypothesis that human and nonhuman animals are indeed sensitive to contingency manipulations and are able to use contingency information as a major hint to infer causal relations. For instance, a scientist will conclude that a given drug produces a skin rash as a side effect if he or she observes that the rash appears often upon consumption of the drug, but does not appear as frequently in the absence of the drug.

The typical paradigm aimed at studying human contingency and causal learning involves exposure to a sequence of trials in which two stimuli-namely a cue (i.e., the potential cause, C) and an outcome (i.e., the potential effect, O) - may or may not co-occur (e.g., Jenkins \& Ward, 1965). The statistical contingency between these stimuli can be manipulated experimentally by determining their joint frequencies. Four combinations are possible with one binary cue and one binary outcome: Both the cue and the outcome may co-occur (type $a$ cell), only the cue may occur (type $b$ cell), only the outcome may occur (type $c$ cell), or neither the cue nor the outcome may occur (type $d$ cell). After the training phase, participants provide a numerical judgment expressing their estimation of the degree to which $\mathrm{C}$ and $\mathrm{O}$ were related to each other. This judgment can then be contrasted with the predictions of descriptive models and normative theories of contingency learning.

One of the most widely used normative standards for the measurement of contingency is the $\Delta P$ index (Allan, 1980; Jenkins \& Ward, 1965). Considering the simplest case, with only one cue and one outcome, each of which can be either present or absent, the $\Delta P$ rule amounts to the difference between the conditional probability of the outcome given that 
the cue is present, $P(\mathrm{O} \mid \mathrm{C})$, and the conditional probability of the outcome given that the cue is absent, $P(\mathrm{O} \mid \neg \mathrm{C})$. Larger differences between the two conditional probabilities provide stronger support for the hypothesis that $\mathrm{C}$ and $\mathrm{O}$ are causally related. When $\Delta P$ equals 0 , no statistical correlation exists between $\mathrm{C}$ and $\mathrm{O}$ and, thus, no apparent reason to believe that they are causally related.

A growing body of evidence has demonstrated that although humans exhibit a remarkable ability to learn accurately from cue-outcome contingency patterns (Shanks \& Dickinson, 1987; Wasserman, 1990), their cognitive systems are easily misled (Alloy \& Abramson, 1979; López, Cobos, Caño, \& Shanks, 1998). This often results in a biased judgment, such as attributing a side effect to a drug that is, in fact, innocuous. As has been discussed elsewhere, these biases have important implications for practical and societal issues, such as the popularity of pseudomedicine (Matute, Yarritu, \& Vadillo, 2011).

Among the factors that have been found to bias human contingency judgments, two are of interest for the purposes of the present report. Even when the contingency is held at zero, manipulations of the probability with which the outcome appears, $P(\mathrm{O})$, may bias judgments such that higher values of $P(\mathrm{O})$ lead to higher contingency judgments. This phenomenon is sometimes referred to as the outcome-density effect (Allan \& Jenkins, 1983; Allan, Siegel, \& Tangen, 2005; Buehner, Cheng, \& Clifford, 2003; Musca, Vadillo, Blanco, \& Matute, 2010; Wasserman, Kao, Van Hamme, Katagiri, \& Young, 1996). In a similar vein, some studies have shown an analogous bias when the probability of the cue, $P(\mathrm{C})$, is manipulated (this is known as the cue-density effect; Allan \& Jenkins, 1983; Matute et al., 2011; Perales, Catena, Shanks, \& González, 2005; Vadillo, Musca, Blanco, \& Matute, 2011; Wasserman et al., 1996). In these cases, the higher the probability of the cue, the higher the subjective judgment of the cue-outcome contingency.

In addition, these biases appear not only in observational situations, in which participants are learning the relationship between two external events (cue and outcome), but also in assessing the relationship between participants' own actions and an outcome. In the latter settings, contingency judgments are also biased by the probability of the outcome (Alloy \& Abramson, 1979; Hannah \& Beneteau, 2009; Matute, 1995; Msetfi, Murphy, \& Simpson, 2007; Msetfi, Murphy, Simpson, \& Kornbrot, 2005; Vallée-Tourangeau, Murphy, \& Baker, 2005) and the probability of the response (which plays the role of the cue in these paradigms; Blanco, Matute, \& Vadillo, 2009, 2011, 2012; Hannah \& Beneteau, 2009; Matute, 1996).

The cue- and outcome-density effects have been proposed as being responsible for many real-life superstitions, illusions, and irrational beliefs in which people perceive a causal relation between two events that do not covary and are not causally related. More specifically, the combination of a high $P(\mathrm{C})$ and a high $P(\mathrm{O})$ has been described as the condition that promotes stronger illusions of causality (e.g., Matute et al., 2011). Consider, for instance, the belief in bogus medicine such as homeopathy: Usually, this erroneous belief appears when treating mild diseases with high chances of spontaneous relief - that is, high $P(\mathrm{O})$ - and when the medicine has no side effects, so that patients can self-administer the medicine very often - that is, high $P(\mathrm{C})$. Nonetheless, an empirical gap renders unclear the question of how certain different combinations of $P(\mathrm{C})$ and $P(\mathrm{O})$ could affect judgments. What would patients' judgments be of the effectiveness of a bogus medicine that is scarcely used (e.g., because it is too expensive or hard to find) in treating a disease whose effects wear off frequently [i.e., low $P(\mathrm{C})$, high $P(\mathrm{O})]$ ? Would these judgments be similar to those in the opposite situation, in which a bogus treatment is used often but the disease rarely disappears [i.e., high $P(\mathrm{C})$, low $P(\mathrm{O})]$ ?

Thus, our present research question concerns the way in which the cue- and outcome-density biases combine when the actual contingency is zero. One possibility is that they simply combine additively. In that case, the stronger contingency overestimation would be observed when the two probabilities are high (i.e., when both the cue and the outcome take place frequently), and this is consistent with the observation of strong beliefs in the effectiveness of actually ineffective treatments (Matute et al., 2011). A second possibility is that the two biases interact, even in an asymmetrical way. In fact, there are reasons to suspect that the two biases may influence contingency judgments unevenly. For instance, although the outcome-density bias seems pervasive and relatively easy to replicate, some authors have described the cue-density effect as elusive and relatively small (Blanco et al., 2011; Hannah \& Beneteau, 2009; Perales \& Shanks, 2007), suggesting the superiority of the former over the latter, at least under certain conditions.

Nonetheless, few studies have addressed the question of whether these two manipulations exert additive or interactive effects on contingency judgments. The few studies that have tested this specific question have typically presented the information to the participants summarized in a tabular format (White, 2004), rather than in the standard trial-bytrial procedure that is normally used in contingency-learning tasks. This difference in the presentation format of the information renders unfair the comparison between the results derived from the two procedures, as has been extensively discussed by many researchers (e.g., Shanks, 1991; ValléeTourangeau, Payton, \& Murphy, 2008). Focusing on the standard trial-by-trial procedure, studies reporting outcomedensity effects in null-contingency settings have generally kept $P(\mathrm{C})$ constant at a medium level (e.g., Msetfi et al., 2007, 2005; Shanks \& Dickinson, 1987). Conversely, studies 
reporting a cue-density effect have been conducted at a high (e.g., Blanco et al., 2009, 2011, 2012; Matute, 1996; Matute et al., 2011) or medium (e.g., Hannah \& Beneteau, 2009; Perales et al., 2005) level of $P(\mathrm{O})$. Therefore, it is unclear whether outcome- or cue-density effects may appear in low $P(\mathrm{C})$ and low $P(\mathrm{O})$ settings, respectively, in the standard contingencylearning task. It could be that a medium-high probability of one of the two stimuli is a necessary condition in order to obtain a density bias by manipulating the probability of the counterpart. This would be consistent with a potential interaction of the two manipulations. This has not been tested in a standard contingency-learning paradigm. The present experiments were designed to fill this empirical gap by manipulating $P(\mathrm{C})$ and $P(\mathrm{O})$, orthogonally and with extreme values (high and low), in a noncontingent task with trial-by-trial presentation format. If potential interactions or asymmetries exist, they should be revealed under these conditions.

\section{Experiment 1}

The aim of Experiment 1 was to clarify the way in which $P(\mathrm{O})$ and $P(\mathrm{C})$ affect contingency judgments. To do so, we manipulated both probabilities in a factorial $2 \times 2$ design while keeping the actual cue-outcome contingency set to zero, as measured by the $\Delta P$ index. It is possible that both manipulations, $P(\mathrm{C})$ and $P(\mathrm{O})$, would produce similar effects, showing an additive relationship when the two probabilities are high. Alternatively, they might interact such that a high, or at least a medium, level of $P(\mathrm{O})$ is necessary in order to show a cue-density effect. The latter possibility has been implicitly assumed in studies that have explored the effects of manipulating $P(\mathrm{C})$ without a low- $P(\mathrm{O})$ condition (Blanco et al., 2009, 2011, 2012; Matute, 1996). Note that the manipulation of the two marginal probabilities in a factorial design while keeping a fixed contingency is possible in a zero-contingency setting only. In Experiment 1, we made use of a causal scenario, which is the standard setting in contingency-learning paradigms.

\section{Method}

Participants and apparatus A group of 108 anonymous Internet users voluntarily participated in the experiment by accessing our virtual laboratory (www.labpsico.deusto.es). The experiment was programmed in JavaScript, a Web-based language that is interpretable by most browsers.

We did not collect demographic information of our sample in our Web-based experiment. Nevertheless, and even though data collected through the Internet can indeed be noisier than data collected in the laboratory, we have previously shown that they are normally consistent with laboratory results and replicate the overestimation-of-contingency effect that has been observed under controlled laboratory conditions (e.g., Matute, Vadillo, Vegas, \& Blanco, 2007). In any case, the basic findings of the present experiment would be replicated in the laboratory in Experiment 2.

The computer program randomly assigned participants to one of the four experimental groups. Two participants were identified as outliers because of their extreme judgments (both $|z| \mathbf{s}>2.70$ ) within their respective groups, and therefore were excluded from the analysis. The resulting sample of 106 participants was distributed across the groups as follows: 27 participants in the HighC-LowO group, 27 in the HighC-HighO group, 26 in the LowC-HighO group, and 26 in the LowC-LowO group. The group names refer to the probabilities (high or low) of the occurrence of the cue, $\mathrm{C}$, and the outcome, $\mathrm{O}$, respectively.

Procedure and design The computer task was an adaptation of the standard allergy paradigm that has been extensively used in the literature on human contingency learning (e.g., Wasserman, 1990). In the present experiment, the participants were asked to imagine that they were medical doctors who had to elucidate whether a fictitious medicine, Batatrim, was effective in helping patients recover from a fictitious illness, "Lindsay Syndrome." Thus, Batatrim was described as a potential cause of the patients' recovery from the disease (see the full instructions in the Appendix).

During the training phase, participants were presented with a series of 100 medical records of fictitious patients suffering from Lindsay Syndrome. In each trial, a message first showed whether the current patient had taken Batatrim, which played the role of the cue or potential cause, C. This information was conveyed using a picture of a pill bottle $(\mathrm{C})$ or the same image crossed out in red $(\neg \mathrm{C})$. Next, the participants predicted whether the patient was going to recover from the illness by clicking on the corresponding button on the screen (labeled "Yes" or "No"). This predictive response was requested to ensure that the participants were reading the information on the screen, as is usually performed in this type of experiment (Vadillo, Miller, \& Matute, 2005). Immediately after the response was recorded, information about the recovery was displayed. The patient either felt better (outcome-present trial, O) or did not (outcome-absent trial, $\neg \mathrm{O}$ ), which was shown by the corresponding pictures (i.e., either a happy face or a sick face). The stimuli for the cues and the outcomes remained on the screen until the participant clicked on the button labeled "Continue" and proceeded to the next trial.

The general design of the experiment is shown in Table 1. In type $a$ trials, both the cue and outcome occurred; in type $b$ trials, the cue occurred but the outcome did not; in type $c$ trials, the outcome occurred but the cue did not; finally, in 
Table 1 Design summary of Experiments 1 and 2

\begin{tabular}{llll}
\hline Group & $P(\mathrm{C})$ & $P(\mathrm{O})$ & Cell Frequencies \\
\hline LowC-LowO & Low $(.20)$ & Low $(.20)$ & $4 a, 16 b, 16 c, 64 d$ \\
LowC-HighO & Low (.20) & High $(.80)$ & $16 a, 4 b, 64 c, 16 d$ \\
HighC-LowO & High (.80) & Low $(.20)$ & $16 a, 64 b, 4 c, 16 d$ \\
HighC-HighO & High (.80) & High $(.80)$ & $64 a, 16 b, 16 c, 4 d$ \\
\hline
\end{tabular}

Group names refer to the two probabilities that were manipulated, $P(\mathrm{C})$ and $P(\mathrm{O})$, whose values are shown in the second and third columns, respectively. The names of the cells (i.e., $a, b, c$, and $d$ ) refer to the standard $2 \times 2$ contingency matrix (see the main text)

type $d$ trials, neither the cue nor the outcome occurred. The probability of taking the medicine, $P(\mathrm{C})$, and the probability of recovery, $P(\mathrm{O})$, were orthogonally manipulated by varying the amounts of $a, b, c$, and $d$ trials during the training session. $P(\mathrm{C})$ and $P(\mathrm{O})$ were fixed to either of two levels, high (.80) or low (.20), resulting in a $2 \times 2$ factorial design comprising four groups. The contingency between taking the medicine and recovering from the illness, as measured by $\Delta P$, was held at zero for all groups.

After the training phase was completed, the participants rated the efficacy of the medicine by answering the question "To what extent do you think that the medicine Batatrim has been effective in healing the crises of the patients you have just seen?" Answers were provided by clicking on a 0-100 numerical scale, where 0 was labeled It was not effective at all and 100 was labeled It was perfectly effective. A floating label appeared as soon as the participants moved their mouse over the scale, indicating the number that was below the mouse pointer. Because the actual contingency between the medicine and the recoveries from the crises was set to zero in all groups, we assumed that a bias had arisen when the participant's judgment departed significantly from zero, suggesting a failure to detect the absence of a relationship between the two stimuli.

\section{Results and discussion}

The critical dependent variable of this experiment was the mean judgment of contingency after the training phase, as is depicted in Fig. 1. These results strongly suggest an interaction between the $P(\mathrm{C})$ and $P(\mathrm{O})$ manipulations. Indeed, a $2 \times 2$ ANOVA yielded significant main effects of both $P(\mathrm{O})$, $F(1,102)=67.80, p<.001, \eta_{\mathrm{p}}{ }^{2}=.40$, and $P(\mathrm{C}), F(1,102)=$ $4.70, p=.033, \eta_{\mathrm{p}}{ }^{2}=.04$, as well as the significant interaction between them, $F(1,102)=4.50, p=.036, \eta_{\mathrm{p}}^{2}=.04$. Further simple-effect analyses showed that a high $P(\mathrm{O})$ was a necessary condition for observing the cue-density bias. When $P(\mathrm{O})$ was high, the group with a high $P(\mathrm{C})$ showed higherthat is, more biased - judgments than did the low- $P(\mathrm{C})$ group, $F(1,102)=9.20, p=.003, \eta_{\mathrm{p}}{ }^{2}=.08$. Consistent with the significant interaction, when $P(\mathrm{O})$ was low, there was no

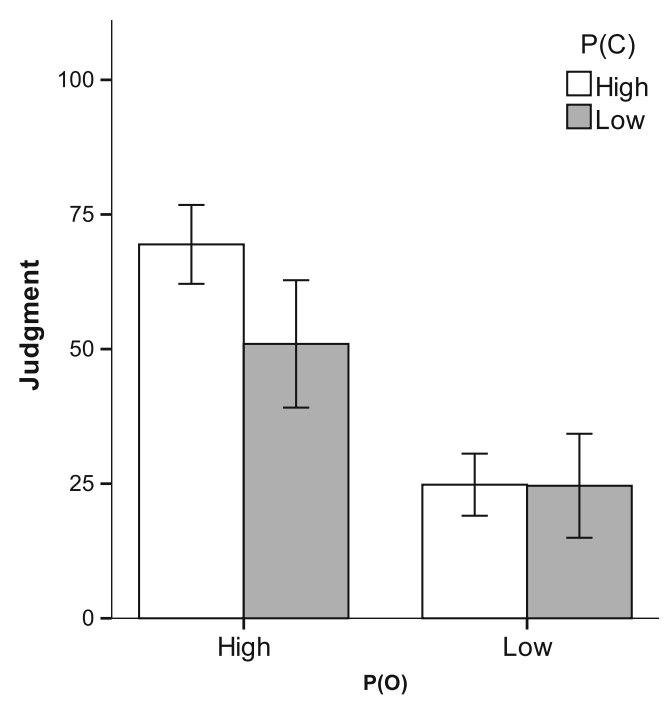

Fig. 1 Mean judgments in Experiment 1, as a function of the probability of the cue, $P(\mathrm{C})$, and the probability of the outcome, $P(\mathrm{O})$. Error bars depict $95 \%$ confidence intervals for the means

difference between groups as a function of $P(\mathrm{C}), F(1,102)$ $<0.01$. In contrast, the outcome-density bias was observed at both levels of $P(\mathrm{C})$ : When $P(\mathrm{C})$ was high, $F(1,102)=54.65$, $p<.001, \eta_{\mathrm{p}}{ }^{2}=.35$, and when it was low, $F(1,102)=18.34$, $p<.001, \eta_{\mathrm{p}}{ }^{2}=.15$. The absence of a cue-density bias in the low- $P(\mathrm{O})$ condition suggests that the significant main effect of cue density was entirely attributable to the high- $P(\mathrm{O})$ condition.

Finally, although the numbers of type $a$ trials were identical in groups HighC-LowO and LowC-HighO (i.e., 16 trials), the judgments were significantly higher in the latter case, in which $P(O)$ was high, $t(51)=4.14, p<.001, d=1.16$. This is also indicative of an interaction between the two probabilities, in which the outcome-density bias seems superior to the cuedensity bias.

\section{Experiment 2}

Two concerns may be put forward against the asymmetry between $P(\mathrm{C})$ and $P(\mathrm{O})$ effects that was observed in Experiment 1. First, it is not possible to disentangle whether the differences between the cue-density and outcome-density effects were, in fact, due to the roles of these stimuli as cue and outcome or to the semantic/perceptual difference between the medicine Batatrim and recovery from the illness. Therefore, in Experiment 2, we used geometric figures rather than causes and effects, and an unfamiliar and neutral cover story that allowed for counterbalancing the two stimuli in their roles as cue and outcome. Second, the absence of differences between groups in the low- $P(\mathrm{O})$ condition might have been due to a floor effect derived from the unidirectional response scale 
(from 0 to 100) that was used in Experiment 1. Participants did not have the opportunity to report a negative contingency and, consequently, may have chosen to give a rating of zero. Thus, if the participants were to answer on a bidirectional scale (from -100 to +100 ), allowing for the report of a perceived negative contingency, then a cue-density bias in the low- $P(\mathrm{O})$ condition might be revealed. In addition, Experiment 2 was run in the laboratory, so as to make sure that the effects observed in Experiment 1 were not due to our use of Internet-based samples.

\section{Method}

Participants and apparatus A group of 166 students from Deusto University volunteered to participate in Experiment 2, which was conducted during four collective sessions in a large computer room at the School of Psychology and Education. The participants were randomly assigned to one of four groups. One participant was excluded because he or she responded "No" to the predictive question in every trial, despite being assigned to the high- $P(\mathrm{O})$ condition, suggesting that he or she was not paying attention to the task. In addition, five participants exhibited extreme judgments $(|z| \mathrm{s}>2.70)$ within their groups, and thus, their data were excluded. The final sample consisted of 160 participants: 41 in the HighC-LowO group, 38 in the HighC-HighO group, 44 in the LowC-LowO group, and 37 in the LowC-HighO group.

Procedure and design The same design was used as in Experiment 1 (see Table 1), but the medical cover story was replaced by a noncausal scenario (see the instructions in the Appendix) in which the participants were asked to learn the predictive relationship between two abstract stimuli (i.e., geometrical figures-namely, a triangle and a square). For roughly half of the participants, the roles of the triangle and the square as cue and outcome were interchanged. For the sake of simplicity, in the following explanation, we will only describe the procedure with the triangle in the role of the cue and the square in the role of the outcome.

On each of the 100 training trials, the occurrence of the cue was indicated by a picture of the triangle and the message "The triangle has appeared," whereas its absence was signaled by the same figure crossed out in red, along with the message "The triangle has not appeared." Next, the participants were asked to predict whether the outcome (i.e., the square) was to occur or not. Upon their providing the dichotomous (yes/no) answer, feedback was displayed by showing either the picture of a square (and the message "The square has appeared") or the square crossed out in red (and the message "The square has not appeared").
The frequency of each type of trial $(a, b, c$, and $d)$ and the randomized sequence of trials were identical to those aspects of Experiment 1. After the training phase, the participants were asked to rate the strength of the predictive relationship between the two figures by answering the question "To what extent do you think that the occurrence of the triangle was useful to predict the occurrence of the square?' Importantly, ratings were provided on a bidirectional scale, ranging from -100 to +100 . As in other experiments using bidirectional scales, the participants were instructed as follows: "A positive number means that the occurrence of the triangle predicts the occurrence of the square. A negative number means that the occurrence of the triangle predicts the absence of the square. A rating of zero means that the triangle cannot be used to predict the square." Numbers from -100 to 100 were shown as participants moved their mouse over the scale.

\section{Results and discussion}

The critical measures were again the contingency judgments at test, shown in Fig. 2. Visual inspection of this figure suggests a replication of the asymmetry that we had found in Experiment 1. The $P(\mathrm{O})$ manipulation seems to have exerted a greater impact on judgments than the $P(\mathrm{C})$ manipulation. A $2 \times 2$ ANOVA, including $P(\mathrm{C})$ and $P(\mathrm{O})$ as factors, was conducted on the judgments. These manipulations did not yield equivalent results. Whereas a significant main effect of $P(\mathrm{O})$ was observed, $F(1,156)=17.58, p<.001, \eta_{\mathrm{p}}{ }^{2}=.10$, the effect of $P(C)$ was not significant, $F(1,156)=1.47, p=$. $23, \eta_{\mathrm{p}}{ }^{2}=.01$. Furthermore, although it was small in size, the interaction between these two factors reached the significance level, $F(1,156)=4.01, p=.047, \eta_{\mathrm{p}}{ }^{2}=.025$.

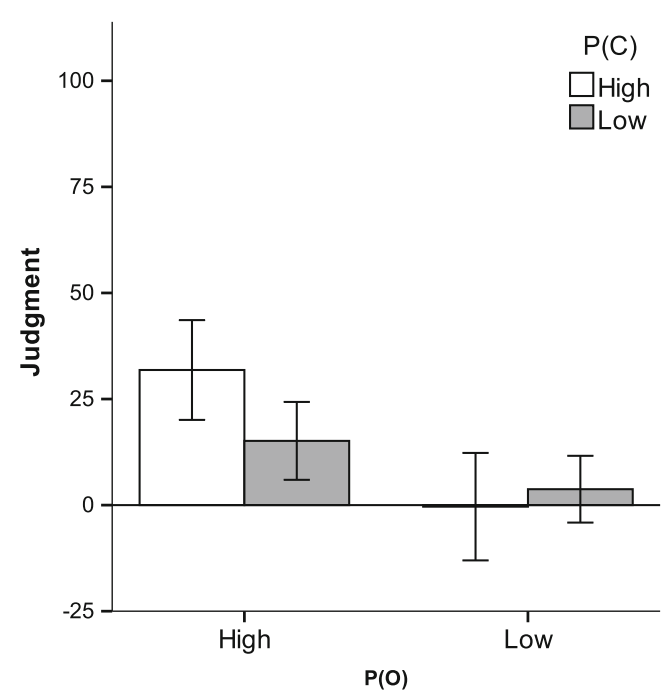

Fig. 2 Mean judgments in Experiment 2, as a function of the probability of the cue, $P(\mathrm{C})$, and the probability of the outcome, $P(\mathrm{O})$. Error bars depict $95 \%$ confidence intervals for the means 
In addition, simple-effect analyses revealed the cue-density effect in the high- $P(\mathrm{O})$ condition, $F(1,156)=4.86, p=.029$, $\eta_{\mathrm{p}}{ }^{2}=.03$, but not in the low- $P(\mathrm{O})$ condition, $F(1,156)<1$. The outcome-density effect was found in the high- $P(\mathrm{C})$ condition, $F(1,156)=19.02, p<.001, \eta_{\mathrm{p}}{ }^{2}=.11$, and was absent in the low- $P(\mathrm{C})$ condition, $F(1,156)=2.42, p=.12, \eta_{\mathrm{p}}{ }^{2}=.02$. As in Experiment 1, the contrast between the two groups with identical numbers of type $a$ trials - that is, HighC-LowO and LowC-HighO - revealed that judgments were higher in the high- $P(\mathrm{O})$ setting, $t(76)=1.97, p=.05, d=0.45$ (although, in Exp. 2, this difference was marginally significant).

Of note, the lack of a cue-density effect in the condition of low probability of the outcome had previously been obtained in Experiment 1. The present replication suggests that this finding was not due to a floor effect artificially produced by the unidirectional response scale used in Experiment 1, as judgments were provided via a bidirectional scale in Experiment 2. This feature of Experiment 2 may also be at the root of the overall small magnitude of the overestimations, as compared to Experiment 1: In Experiment 2, half of the screen was occupied by the positive values of the scale only, while in Experiment 1, the same space was occupied by positive and high (i.e., larger than 50) values.

In summary, the results of Experiment 2 showed that the cue- and outcome-density effects appeared prominently against a background of high $\mathrm{P}(\mathrm{O})$ and high $\mathrm{P}(\mathrm{C})$, respectively. The reported statistical interaction revealed that the biases were less likely to appear in the low- $P(\mathrm{C})$ and low- $P(\mathrm{O})$ conditions. Finally, while the main effect of outcome density was reliable, the main effect of the $P(\mathrm{C})$ manipulation failed to reach the significance level, suggesting that the impact of the latter manipulation is somewhat weaker. These conclusions align with those of Experiment 1 and support the argument that the effects of manipulating $P(\mathrm{C})$ and $P(\mathrm{O})$ on contingency judgments are asymmetrical and interactive. Furthermore, a combined analysis of the two experiments, including Experiment, $P(\mathrm{C})$, and $P(\mathrm{O})$ as factors and taking the $z$ scores of the judgments as the dependent variable, revealed results similar to those conducted on each experiment separately: We found a main effect of $P(\mathrm{O}), F(1,258)=70.80, p<.001, \eta_{\mathrm{p}}{ }^{2}=.22$, a main effect of $P(\mathrm{C}), F(1,258)=5.23, p=.023, \eta_{\mathrm{p}}{ }^{2}=.02$, and their interaction, $F(1,258)=7.81, p=.006, \eta_{\mathrm{p}}{ }^{2}=.03$, indicating that the cue-density bias was observable under high- $P(\mathrm{O})$ conditions only, $F(1,258)=12.61, p<.001, \eta_{\mathrm{p}}{ }^{2}=.05$. In addition, a two-way $P(\mathrm{O}) \times$ Experiment interaction showed that the main outcome-density bias was more pronounced in Experiment 1 than in Experiment 2, consistent with the abovementioned observation about the rating scale.

Several additional controls and improvements over Experiment 1 were introduced in Experiment 2. First, the semantic/perceptual features of cues and outcomes were controlled (by counterbalancing them and using a noncausal, abstract cover story involving geometrical figures). Second, a bidirectional scale was utilized to ensure that no floor effects artificially prevented the cue-density bias in the low- $P(\mathrm{O})$ condition. Third, whereas the Experiment 1 data were collected through the Internet, the Experiment 2 data were collected in a laboratory. Finally, Experiment 2 allows for the generalization of the findings of Experiment 1 to a noncausal scenario in which there is little or no room for prior knowledge about the way in which cues and outcomes should relate to each other.

\section{General discussion}

The present two experiments provide further evidence that human contingency judgments are easily biased by covariational manipulations, often departing from the objective contingency values provided by normative indexes such as $\Delta P$ (see, e.g., Allan \& Jenkins, 1983; Alloy \& Abramson, 1979; López et al., 1998). The frequencies of both the cue and the outcome occurrences were able to produce overestimations of contingency (i.e., cue- and outcome-density biases). Moreover, these two manipulations seemed to interact. Particularly, we have shown that the overestimation of contingency is magnified when both the cue and the outcome are presented very frequently, and it is weak or disappears when one of the two stimuli is presented infrequently.

In fact, as we noted in the introduction, many of the previous experimental studies that reported cue- and outcome-density biases had used a high- $P(\mathrm{O})$ condition (e.g., Blanco et al., 2011, 2012; Hannah \& Beneteau, 2009; Matute, 1996; Matute et al., 2011; Perales et al., 2005) or a medium/high- $P(C)$ condition (e.g., Msetfi et al., 2007, 2005), respectively. Our results complement these studies, because they provide evidence for the weakening of the biases in backgrounds of low $P(\mathrm{O})$ and low $P(\mathrm{C})$.

In addition, taking together the results of Experiments 1 and 2, we can suggest that the outcome-density bias is less vulnerable to this interactive combination than is the cuedensity bias, because the main effect of the $P(\mathrm{C})$ manipulation was obtained only in Experiment 1, and as we discussed above, it can be completely attributed to the high- $P(\mathrm{O})$ condition, which boosted the cue-density bias. By contrast, the main effect of the $P(\mathrm{O})$ manipulation was readily obtained and exhibited larger effects in the two experiments, although it was, again, stronger against a background of high $P(\mathrm{C})$.

The demonstration of an interactive effect of the $P(\mathrm{C})$ and $P(\mathrm{O})$ manipulations is not only an empirical question that deserves being studied, but also entails some implications for the theoretical debate. Models of contingency learning should be able to accommodate the present results. Much has been discussed on the mechanism at the root of the 
biases in contingency judgments. Some have argued (e.g., Allan, 1980) that these biases are led by contiguity, or the number of cue-outcome co-occurrences (i.e., of type $a$ cells). Then, because the manipulations of $P(\mathrm{C})$ and $P(\mathrm{O})$ here were mutually independent, both should contribute evenly to the probability of co-occurrences. In other words, if the biases were produced by the number of type $a$ cells, one would expect an additive, rather than an interactive, combination of the two manipulations, $P(\mathrm{C})$ and $P(\mathrm{O})$. In fact, our finding of interactive, asymmetrical effects of these manipulations on the judgments suggests that their contributions to the overestimation of contingency are uneven. In addition, the overestimation cannot be fully explained by the number of type $a$ cells because, when we compared the two groups in which the numbers of this type of trial were identical, we found stronger illusions when $P(\mathrm{O})$ was higher, in both experiments.

A more refined account of these biases would not rely on type $a$ trials solely. Indeed, many researchers have argued that people weight differently each piece of information when judging contingencies (e.g., Perales \& Shanks, 2007; White, 2004). Interestingly, the ranking of cell weights that has usually been reported in previous research (i.e., $a>b>$ $c>d$; see Kao \& Wasserman, 1993; Wasserman, Dorner, \& Kao, 1990; Wasserman et al., 1996) could be used to predict our present results. This ranking can be incorporated in several contingency-learning models (e.g., the EI model [Perales \& Shanks, 2007] or the Rescorla-Wagner model [Rescorla \& Wagner, 1972]). Although our present experiments are, admittedly, unable to conclusively discriminate between specific theories, they do suggest that any model that does not incorporate, either explicitly (e.g., White, 2004) or implicitly (e.g., Rescorla \& Wagner, 1972), unequal cell weights will face problems in offering a complete account of the cue- and outcome-density effects.

Importantly, the relevance of cue- and outcome-density biases is not restricted to the academic debate. As has been discussed elsewhere, both biases have important implications for the study of everyday causal illusions and superstitions (e.g., Matute et al., 2011), as well as stereotype formation (e.g., Murphy, Schmeer, Vallée-Tourangeau, Mondragón, \& Hilton, 2011). The knowledge obtained from studying overestimations of null contingency should, therefore, be of great value in the development of strategies to reduce the impact of irrationality in modern society (see, e.g., Lewandowsky, Ecker, Seifert, Schwarz, \& Cook, 2012; Lilienfeld, Ammirati, \& Landfield, 2009; Matute et al., 2011).

Author Note Support for this research was provided by the Dirección General de Investigación of the Spanish Government (Grant No. PSI2011-26965) and the Departamento de Educación, Universidades e Investigación of the Basque Government (Grant No. IT363-10). We thank Samuel Hannah for his helpful comments on an earlier version of this article.

\section{Appendix}

Full instructions of Experiment 1 (Translated from the original in Spanish)

Imagine that you are a doctor who works at the Hospital Emergency Department. You are a specialist in a rare and dangerous disease called "Lindsay Syndrome," which must be treated quickly in the emergency room.

Crises induced by this illness may be stopped immediately by using a medicine called "Batatrim," but this medicine is still in its testing stage, and therefore its reliability has not been yet proven. In addition, you should know that it produces several side effects, which can be severe, in every patient who takes it, so it cannot always be used.

When you click on the "Start" button, you will be presented with a series of medical records of patients suffering from Lindsay Syndrome. In each record, you will first know whether or not the patient was given Batatrim, and you will be asked to indicate whether you think that the patient will recover from the crisis.

Then, you will know whether or not the patient actually recovered from the crisis. Try to find out the extent to which Batatrim is actually effective. Once you see a number of patients, you will be asked a few questions.

Full instructions of Experiment 2 (Translated from the original in Spanish)

In this experiment, two geometrical figures (a square and a triangle) will appear on your computer screen.

In each trial, you will first know whether or not the square [the triangle] appears (when it is does not appear, it will be crossed out in red). You will be asked to predict whether the other figure, the triangle [the square], will appear, by clicking on the corresponding button.

Once you make your prediction, you will know whether the triangle [square] actually appeared. Your goal is to try to find out the extent to which the square [triangle] can help you predict when the triangle [square] will appear.

\section{References}

Allan, L. G. (1980). A note on measurement of contingency between two binary variables in judgment tasks. Bulletin of the Psychonomic Society, $15,147-149$.

Allan, L. G., \& Jenkins, H. M. (1983). The effect of representations of binary variables on judgment of influence. Learning and Motivation, 14, 381-405.

Allan, L. G., Siegel, S., \& Tangen, J. M. (2005). A signal detection analysis of contingency data. Learning \& Behavior, 33, 250-263. doi:10.3758/BF03196067

Alloy, L. B., \& Abramson, L. Y. (1979). Judgment of contingency in depressed and nondepressed students: Sadder but wiser? Journal of 
Experimental Psychology. General, 108, 441-485. doi:10.1037/ 0096-3445.108.4.441

Blanco, F., Matute, H., \& Vadillo, M. A. (2009). Depressive realism: Wiser or quieter? Psychological Record, 59, 551-562.

Blanco, F., Matute, H., \& Vadillo, M. A. (2011). Making the uncontrollable seem controllable: The role of action in the illusion of control. Quarterly Journal of Experimental Psychology, 64, 1290-1304. doi:10.1080/17470218.2011.552727

Blanco, F., Matute, H., \& Vadillo, M. A. (2012). Mediating role of the activity level in the depressive realism effect. PLOS ONE, 7, e46203. doi:10.1371/journal.pone. 0046203

Buehner, M. J., Cheng, P. W., \& Clifford, D. (2003). From covariation to causation: A test of the assumption of causal power. Journal of Experimental Psychology: Learning, Memory, and Cognition, 29, $1119-1140$

Hannah, S., \& Beneteau, J. L. (2009). Just tell me what to do: Bringing back experimenter control in active contingency tasks with the command-performance procedure and finding cue-density effects along the way. Canadian Journal of Experimental Psychology, 63, 59-73.

Hume, D. (1987). A treatise of human nature (2nd ed.). Oxford: Clarendon Press.

Jenkins, H. M., \& Ward, W. C. (1965). Judgement of contingency between responses and outcomes. Psychological Monographs, 79, 1-17.

Kao, S.-F., \& Wasserman, E. A. (1993). Assessment of an information integration account of contingency judgment with examination of subjective cell importance and method of information presentation. Journal of Experimental Psychology: Learning, Memory, and Cognition, 19, 1363-1386.

Lewandowsky, S., Ecker, U. K. H., Seifert, C. M., Schwarz, N., \& Cook, J. (2012). Misinformation and its correction: Continued influence and successful debiasing. Psychological Science in the Public Interest, 13, 106-131.

Lilienfeld, S. O., Ammirati, R., \& Landfield, K. (2009). Giving debiasing away: Can psychological research on correcting cognitive errors promote human welfare? Perspectives on Psychological Science, 4, 390-398.

López, F. J., Cobos, P. L., Caño, A., \& Shanks, D. R. (1998). The rational analysis of human causal and probability judgment. In M. Oaksford \& N. Chater (Eds.), Rational models of cognition (pp. 314-352). Oxford: Oxford University Press.

Matute, H. (1995). Human reactions to uncontrollable outcomes: Further evidence for superstitions rather than helplessness. Quarterly Journal of Experimental Psychology, 48, 142-157.

Matute, H. (1996). Illusion of control: Detecting response-outcome independence in analytic but not in naturalistic conditions. Psychological Science, 7, 289-293.

Matute, H., Vadillo, M. A., Vegas, S., \& Blanco, F. (2007). Illusion of control in Internet users and college students. Cyberpsychology \& Behavior, 10, 176-181. doi:10.1089/cpb.2006.9971

Matute, H., Yarritu, I., \& Vadillo, M. A. (2011). Illusions of causality at the heart of pseudoscience. British Journal of Psychology, 102, 392-405.

Msetfi, R. M., Murphy, R. A., \& Simpson, J. (2007). Depressive realism and the effect of intertrial interval on judgements of zero, positive, and negative contingencies. Quarterly Journal of Experimental Psychology, 60, 461-481.

Msetfi, R. M., Murphy, R. A., Simpson, J., \& Kornbrot, D. E. (2005). Depressive realism and outcome density bias in contingency judgments: The effect of the context and intertrial interval. Journal of Experimental Psychology. General, 134, 10-22.
Murphy, R. A., Schmeer, S., Vallée-Tourangeau, F., Mondragón, E., \& Hilton, D. (2011). Making the illusory correlation effect appear and then disappear: The effects of increased learning. Quarterly Journal of Experimental Psychology, 64, 24-40.

Musca, S. C., Vadillo, M. A., Blanco, F., \& Matute, H. (2010). The role of cue information in the outcome-density effect: Evidence from neural network simulations and a causal learning experiment. Connection Science, 22, 177-192.

Perales, J. C., Catena, A., Shanks, D. R., \& González, J. A. (2005). Dissociation between judgments and outcome-expectancy measures in covariation learning: A signal detection theory approach. Journal of Experimental Psychology: Learning, Memory, and Cognition, 31, 1105-1120.

Perales, J. C., \& Shanks, D. R. (2007). Models of covariation-based causal judgments: A review and synthesis. Psychonomic Bulletin \& Review, 14, 577-96.

Rescorla, R. A. (1968). Probability of shock in the presence and absence of CS in fear conditioning. Journal of Comparative and Physiological Psychology, 66, 1-5.

Rescorla, R. A., \& Wagner, A. R. (1972). A theory of Pavlovian conditioning: Variations in the effectiveness of reinforcement and nonreinforcement. In A. H. Black \& W. F. Prokasy (Eds.), Classical conditioning II: Current research and theory (pp. 6499). New York: Appelton-Century-Crofts.

Shanks, D. R. (1991). On similarities between causal judgments in experienced and described situations. Psychological Science, 2, $341-350$

Shanks, D. R., \& Dickinson, A. (1987). Associative accounts of causality judgment. In G. H. Bower (Ed.), The psychology of learning and motivation, Vol. 21: Advances in research and theory (pp. 229-261). San Diego: Academic Press.

Vadillo, M. A., Miller, R. R., \& Matute, H. (2005). Causal and predictive-value judgments, but not predictions, are based on cue-outcome contingency. Learning \& Behavior, 33, 172-183. doi:10.3758/BF03196061

Vadillo, M. A., Musca, S. C., Blanco, F., \& Matute, H. (2011). Contrasting cue-density effects in causal and prediction judgments. Psychonomic Bulletin \& Review, 18, 110-115. doi:10.3758/ s13423-010-0032-2

Vallée-Tourangeau, F., Murphy, R. A., \& Baker, A. G. (2005). Contiguity and the outcome density bias in action-outcome contingency judgements. Quarterly Journal of Experimental Psychology, 58(2), 177-192.

Vallée-Tourangeau, F., Payton, T., \& Murphy, R. A. (2008). The impact of presentation format on causal inferences. European Journal of Cognitive Psychology, 20, 177-194.

Wasserman, E. A. (1990). Detecting response-outcome relations: Toward an understanding of the causal texture of the environment. In G. H. Bower (Ed.), The psychology of learning and motivation (Vol. 26, pp. 27-82). San Diego: Academic Press.

Wasserman, E. A., Dorner, W. W., \& Kao, S.-F. (1990). Contributions of specific cell information to judgments of interevent contingency. Journal of Experimental Psychology: Learning, Memory, and Cognition, 16, 509-521.

Wasserman, E. A., Kao, S.-F., Van Hamme, L. J., Katagiri, M., \& Young, M. E. (1996). Causation and association. In D. R. Shanks, K. J. Holyoak, \& D. L. Medin (Eds.), The psychology of learning and motivation (Causal learning, Vol. 34, pp. 207-264). San Diego: Academic Press.

White, P. A. (2004). Causal judgment from contingency information: A systematic test of the pCI rule. Memory \& Cognition, 32, 353-368. 